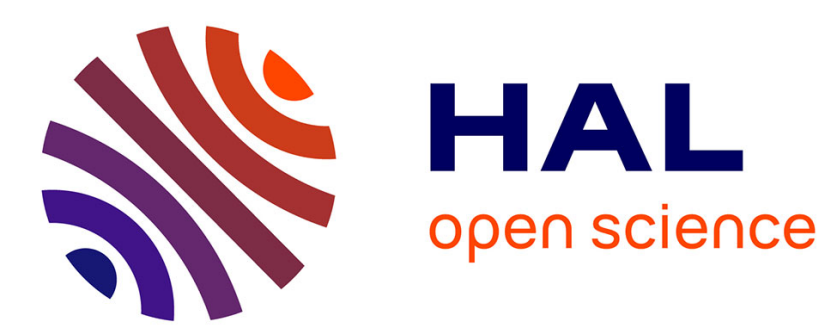

\title{
Magnetic nanoparticles and their applications in medicine
}

Etienne Duguet, Sébastien Vasseur, Stéphane Mornet, Jean-Marie Devoisselle

\section{To cite this version:}

Etienne Duguet, Sébastien Vasseur, Stéphane Mornet, Jean-Marie Devoisselle. Magnetic nanoparticles and their applications in medicine. Nanomedicine, 2006, 1 (2), pp.157-168. 10.2217/17435889.1.2.157 . hal-00090680

\section{HAL Id: hal-00090680 \\ https://hal.science/hal-00090680}

Submitted on 3 May 2021

HAL is a multi-disciplinary open access archive for the deposit and dissemination of scientific research documents, whether they are published or not. The documents may come from teaching and research institutions in France or abroad, or from public or private research centers.
L'archive ouverte pluridisciplinaire HAL, est destinée au dépôt et à la diffusion de documents scientifiques de niveau recherche, publiés ou non, émanant des établissements d'enseignement et de recherche français ou étrangers, des laboratoires publics ou privés. 


\title{
Magnetic nanoparticles and their applications in medicine
}

\author{
Etienne Duguet ${ }^{1 \dagger}$, \\ Sébastien Vasseur ${ }^{l}$, \\ Stéphane Mornet ${ }^{1}$ \& \\ Jean-Marie Devoisselle ${ }^{2}$ \\ ${ }^{\dagger}$ Author for correspondence \\ ${ }^{1}$ Institut de Chimie de la \\ Matière Condensée de \\ Bordeaux, CNRS/University \\ Bordeaux-1, 87 avenue du Dr \\ Albert Schweitzer, F-33608 \\ Pessac Cedex, France \\ Tel.: +33 540002651 ; \\ Fax: +33540002761 ; \\ E-mail:duguet@ \\ icmcb-bordeaux.cnrs.fr \\ ${ }^{2} U M R$ CNRS/ENSCM/ \\ UM1 5618, Institut Charles \\ Gerhardt, 8, rue de I'Ecole \\ Normale, F-34296 \\ Montpellier Cedex 5, France
}

Keywords: cancer, drug delivery, hyperthermia, magnetic guidance, magnetic nanoparticle, MRI contrast agent, stealthiness, targeting

\begin{abstract}
Magnetic nanoparticles have attracted attention in modern medicine and pharmacology owing to their potential usefulness as contrast agents for MRI, as colloidal mediators for cancer magnetic hyperthermia or as active constituents of drug-delivery platforms. This review examines these in vivo applications through an understanding of the involved problems and the current and future possibilities for resolving them. A special emphasis is placed upon magnetic nanoparticle requirements from a physical viewpoint (e.g., relaxivity for MRI, specific absorption rate for hyperthermia and magnetic guidance), the factors affecting their biodistribution after intravenous injection (e.g., size and surface hydrophobic/hydrophilic balance) and the solutions envisaged for enhancing their half-life in the blood compartment and in targeting tumor cells.
\end{abstract}

Magnetism and medicine have shared a fascinating common history for several tenths of centuries [1]. In recent decades, the miniaturization of electromagnets, the development of superconducting electromagnets and the introduction of strong permanent magnets ( $\mathrm{Sm}-\mathrm{Co}$ and $\mathrm{Nd}-\mathrm{Fe}-\mathrm{B}$ ) have allowed the medical use of magnets to spread to fields as diverse as dentistry, cardiology, neurosurgery, oncology and radiology.

Moreover, well calibrated submicrometric particles are currently produced in large amounts thanks to reproducible synthetic protocols and several chemical routes, allowing their surface to be efficiently modified to ensure their colloidal stability in liquids, protect them from corrosion/dissolution and/or derivatizing with biorelated molecules. Nanoparticles with controllable sizes ranging from a few nanometers up to tens of nanometers are of particular interest (Table 1). First, they are smaller than cells and comparable with viruses, proteins and genes. Therefore, they are able to cross biological membranes and interact closely with biomolecules. Second, unusual physical properties may result from so divided materials, such as quantum confinement in semiconductor materials (bright and narrow fluorescence of quantum dots) [2], dielectric confinement in gold colloids (plasmon resonance) [3] and superparamagnetism in magnetic materials. Thus, nanoparticles became recently inescapable tools for biologists, pharmacologists and physicians. A special development effort concerned magnetic nanoparticles, which are now used routinely as contrast agents in MRI and whose next applications are expected in the field of cancer thermal treatment, magnetic targeting and remotely triggered drug release.

Nevertheless, the use of magnetic nanoparticles in the blood compartment depends on specific requirements with respect to their plasma half-life and final biodistribution. The problem of the non-natural stealthiness of the nanoparticles towards the immune system and the possibilities for resolving it have been studied widely in the field of drug delivery from liposomes and polymeric nanoparticles [4]. Indeed, retention of drugs in the circulation is a key step in the design of drug delivery devices. Even the most active compound in vitro is useless if it does not reside in vivo in the blood long enough to reach its target, while managing to avoid, to some extent, premature metabolism, immunological reactions, toxicity, rapid excretion and captation by undesired tissues [5]. Presently, many pieces of information are available concerning the immune system mechanisms, the factors affecting the biodistribution of polymeric nanoparticles, such as their size and shape, the hydrophobic/hydrophilic balance of their surface, their surface charge and the solutions envisaged for targeting specific organs or tumor cells.

This fast-emerging research field was recently reviewed from a general point of view [6-10] and on the basis of nanoparticle synthesis [11,12] or physical properties [13].

The aim of this review is to present an updated description of the potential of magnetic nanoparticles for in vivo applications through an understanding of the problems involved from the 
Table 1. Main nanoparticles developed for biomedical applications.

\begin{tabular}{|c|c|c|c|}
\hline Core type & Examples & Size $(\mathrm{nm})$ & $\begin{array}{l}\text { Current and/or future } \\
\text { biomedical applications }\end{array}$ \\
\hline \multicolumn{4}{|l|}{ Organic } \\
\hline Liposomes & Based on phospholipids & $50-500$ & $\begin{array}{l}\text { Drug carriers potentially thermo-, photo- or } \\
\mathrm{pH} \text {-sensitive }[18,20,85-91]\end{array}$ \\
\hline Micelles & $\begin{array}{l}\text { Based on surfactants or } \\
\text { block copolymers }\end{array}$ & $<50$ & $\begin{array}{l}\text { Drug carriers potentially thermo-, photo- or } \\
\text { pH-sensitive }[18,20,85-91]\end{array}$ \\
\hline $\begin{array}{l}\text { Biodegradable polymeric } \\
\text { capsules or spheres }\end{array}$ & $\begin{array}{l}\text { Polylactide, poly(lactic-co- } \\
\text { glycolic acid) and } \\
\text { polyalkylcyanoacrylate }\end{array}$ & $10-1000$ & $\begin{array}{l}\text { Drug carriers potentially thermo-, photo- or } \\
\text { pH-sensitive }[18,20,85-91]\end{array}$ \\
\hline \multicolumn{4}{|l|}{ Inorganic } \\
\hline $\begin{array}{l}\text { Semiconductor } \\
\text { quantum dots }\end{array}$ & $\mathrm{CdS}$ & $2-8$ & Fluorescent probes for optical diagnosis [2] \\
\hline Noble metal colloids & Gold and silver & $2-20$ & $\begin{array}{l}\text { Fluorescent probes for optical diagnosis; } \\
\text { light-guided and light-activated therapies [3] }\end{array}$ \\
\hline Magnetic nanoparticles & $\begin{array}{l}\text { Maghemite } \gamma-\mathrm{Fe}_{2} \mathrm{O}_{3} \text {, } \\
\text { magnetite } \mathrm{Fe}_{3} \mathrm{O}_{4} \text {, iron, } \\
\text { cobalt and nickel }\end{array}$ & $5-50$ & $\begin{array}{l}\text { MRI contrast agents; hyperthermia mediators; } \\
\text { magnetically targeted drug carriers [6-13] }\end{array}$ \\
\hline
\end{tabular}

viewpoint of their overall requirements, their synthesis and their bulk and surface properties. The main intention of this contribution is to impart information concerning both the state of the art as well as the need for further progress and clinical development. Only typical examples will be reported and discussed and a special emphasis will be given to cancer diagnosis and therapy.

\section{Magnetic nanoparticle design}

Magnetic nanoparticles may be of different chemical compositions. Iron oxide particles, such as magnetite $\mathrm{Fe}_{3} \mathrm{O}_{4}$ or its oxidized (and hence more stable) form maghemite $\gamma-\mathrm{Fe}_{2} \mathrm{O}_{3}$, are by far the most commonly employed oxides for biomedical applications [11]. The main factors are their good chemical stability and their biocompatibility. Metal nanoparticles (e.g., cobalt and nickel) are more powerful from the viewpoint of their magnetic properties, but they are toxic and, hence, cannot be envisaged for clinical applications. Metal iron could be used, however, because of the large surface area of nanoparticles, it is spontaneously converted to iron oxyhydroxide in aqueous media.

\section{Synthesis, colloidal stabilization in} physiological medium \& magnetic properties Even if several routes to prepare iron oxide nanoparticles exist [11], the most commonly used method is based on the alkaline coprecipitation of iron (II) and iron (III) precursors in aqueous solutions. It is generally conceded that, at the nanoparticle surface, the crystal has hydroxyl groups at its end, which interact strongly with physisorbed water layers through hydrogen bonding. Such hydroxyl groups present acidic and basic properties leading to protonation at low $\mathrm{pH}$ values and deprotonation at high $\mathrm{pH}$ values, resulting in positively and negatively charged surfaces, respectively. In both conditions, nanoparticle surfaces repel themselves, preventing or delaying particle aggregation (electrostatic stabilization). The aqueous dispersions are stable for long periods and, when the nanoparticle concentration is high enough, they act as ferrofluids. However, at physiological $\mathrm{pH}$, the surface charge density is too low (isoelectric point at $\mathrm{pH} 7$ for iron oxides) and therefore the nanoparticles are aggregated in the form of flocculates and, thus, cannot be administered. Therefore, the only way to stabilize iron oxide nanoparticles in water is to ensure their steric stabilization by decorating their surface with long hydrophilic (macro)molecules that act as a repellent coating.

Among the large family of magnetic materials, ferro- or ferri-magnetic materials and superparamagnetic materials are of special interest in biomedical applications [14]. The first class is found in massive materials or submicronic particles: the susceptibility is large, the magnetization increases massively with the magnetic field intensity, a remnant magnetization occurs after removal of the magnetic field and, above a critical temperature called the Curie temperature (Tc), the ferroor ferri-magnetic behavior disappears. The largest 
iron oxide nanoparticles belong to this class (e.g., $\mathrm{Tc}=580^{\circ} \mathrm{C}$ for $\mathrm{Fe}_{3} \mathrm{O}_{4}$ ). If their size is lower than approx 10-20 nm, iron oxide nanoparticles are said to be 'superparamagnetic' and their main feature is the absence of remnant magnetization, limiting magnetic interactions within particles and, therefore, facilitating their stabilization as ferrofluids in aqueous solutions.

\section{Size/surface requirements with regard to intravenous administration}

Intravenous (i.v.) administration is the most effective method of reaching target organs and tissues since all the vital cells receive supply through the blood circulation. The fundamental question regarding nanoparticle fate in the blood compartment has been discussed widely by all the physicians and scientists involved in the design and the development of devices for controlled drug delivery (i.e., essentially liposomes and polymeric particles) [5]. As soon as bare particles are intentionally or unintentionally injected into the blood compartment, they become public enemy number one of the mononuclear phagocyte system (MPS) [15,16]. The first step of the clearance mechanism is the spontaneous adsorption of plasma proteins (opsonization process), which are capable of interacting with the specialized plasma membrane receptors on monocytes and macrophages, thus promoting particle recognition by these cells (Figure 1). The second step consists of the endocytosis/phagocytosis of the particles by the circulating monocytes or the fixed macrophages, leading to their elimination from circulation and their simultaneous concentration in organs with high phagocytic activity. Thus, after i.v. administration, the particles are cleared within minutes from the bloodstream and their typical final biodistribution is approximately $80-90 \%$ in liver, $5-8 \%$ in spleen and $1-2 \%$ in bone marrow [5]. The passive targeting strategy takes advantage of such an inescapable fate of particles for delivering drug or contrast agents to MPS organs [17] or infection/inflammation areas where macrophage activity is high [18].

If monocytes and macrophages in contact with blood are not the desired target, a strategy of active targeting must be developed and its first requirement consists of minimizing or delaying nanoparticle uptake by the MPS [15]. The most satisfactory strategy consists of designing macrophage-evading nanoparticles, also called long-circulating nanoparticles or stealth nanoparticles. Among the physicochemical factors that are known to have an effect on the opsonization process, the size, surface charge density and hydrophilicity/hydrophobicity balance have been studied widely, either in liposome or polymeric nanoparticle systems [15]. The main conclusion is that the smaller, more neutral and more hydrophilic the nanoparticle surface, the longer its plasma half-life. Concerning the size effect, this indicates that surface curvature changes may affect the extent and/or type of opsonin adsorption. It is generally assumed that surface features are more important than those of the core, because the surface contacts blood and organs directly [19]. For hydrophobic particles, many studies have concerned the development of corecorona structures, where the corona is made of hydrophilic macromolecules for creating polymer brushes, acting as a steric surface barrier and reducing opsonin adsorption. Among the natural or artificial macromolecules, linear dextrans and derivatives are widely used. Among the synthetic ones, poly(ethylene glycol) (PEG) is very probably the most popular ('PEGylation' technique) [20]. PEG is the most effective polymer for suppressing protein adsorption. The optimal molecular weight varies between 2000 and $5000 \mathrm{~g} / \mathrm{mol}$ $[15,21]$, but the surface density of macromolecules is also a critical parameter. It should be noted that, concerning iron oxide nanoparticles, the hydrophilic macromolecular corona plays the roles of steric stabilizer and stealthiness agent simultaneously [22].

Lastly, long-circulating particle escape from the circulation is normally restricted to sites where the capillaries have opened fenestrations, as in the sinus endothelium of the liver or when the integrity of the endothelial barrier is perturbed by inflammatory processes (e.g., rheumatoid arthritis, infarction and infections) or by some types of tumors [15]. Therefore, the idea of exploiting such vascular abnormalities for extravasating and accumulating nanoparticles in these inflammatory sites or tumors is also particularly attractive. Such a strategy is also considered to be passive targeting, apart from the MPS mediation.

Beyond stealthiness, ligand conjugation is the second requirement for active targeting. Such ligands are expected to bind specifically to surface epitopes or receptors on the target sites. Such a strategy should open the possibility of targeting specific cell types or subsets of cells within the vasculature and even elements of vascular emboli and thrombi [15]. In the case of cancer diagnosis or therapy, active targeting could allow the selective targeting of cancer cells, even if they have escaped the tumor mass and disseminated as metastatic cells. 
Figure 1. The main steps of the mononuclear phagocyte system-mediated clearance of nonstealth particles.

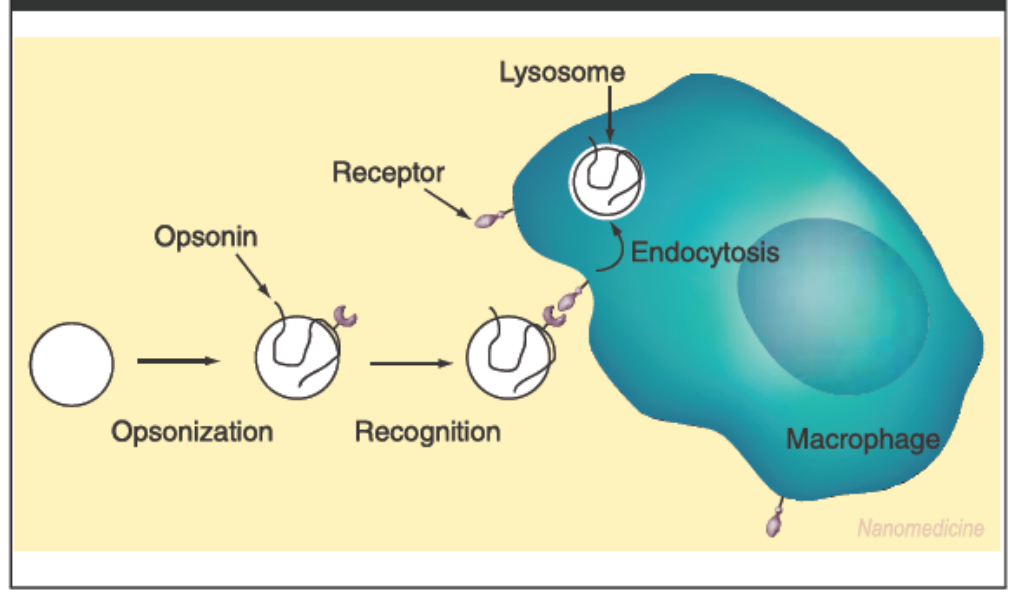

These ligands include oligosaccharides, oligopeptides, folic acid, antibodies and their fragments. The coupling of monoclonal antibodies has at least two disadvantages: the overall dimensions of the antibodies $(\sim 20 \mathrm{~nm})$, which cause particles to diffuse poorly through biological barriers, and their immunogenicity. Therefore, the coupling of antibody fragments or small nonantigenic ligands to nanoparticles has been also investigated, for example, folic acid (B vitamin essential for cell division processes) for tumor targeting [23].

\section{Magnetic nanoparticles as contrast agents for MRI}

In actual practice, tissues may be differentiated on MR images. But, in many clinical situations, these intrinsic differences are small and exogenous contrast media are currently used for a better delineation of tissues. The first generation of these contrast agents consisted of high-spin paramagnetic ions, usually $\mathrm{Gd}^{3+}$ in very stable nontoxic chelate form obtained through complexation by low molecular weight chelating molecules, such as diethylenetriaminepentaacetic acid. Gd-chelates have a nonselective extracellular distribution before their excretion by the kidney. Hydrogen atoms of water in proximity to such chelates experience a faster $\mathrm{T}_{1}$-relaxation. Consequently, differences in agent concentration result in contrast enhancement on $\mathrm{T}_{1}$-weighted images ('positive' contrast) [6].

Magnetic nanoparticles have also been developed as contrast agents for both standard and functional MRI [24,25]. The superparamagnetic behavior of these subdomain magnetic cores is similar to that of paramagnetic substances, in that they lose their magnetization when the magnetic field is removed, but differs by the value of the magnetic moment, which is markedly higher. Therefore, their relaxivity (ability to increase the relaxation rates of surrounding proton spins) is much higher than that of Gd-chelates. In most situations, they are used for their significant capacity to produce predominantly $\mathrm{T}_{2}$-relaxation effects, which result in signal reduction on $\mathrm{T}_{2}$ weighted images ('negative' contrast). Basically, the phenomenon may be described from the large magnetic field heterogeneity around the nanoparticle through which water molecules diffuse [26]. Diffusion induces dephasing of the proton magnetic moments, resulting in $\mathrm{T}_{2}$ shortening. Such contrast agents are also called susceptibility agents owing to their effect on the magnetic field. $\mathrm{T}_{2}$-shortening is a remote effect, whereas the $T_{1}$-shortening process requires a close interaction between water molecules and Gd-chelates [6].

This new generation of contrast agent is often called (ultrasmall) superparamagnetic iron oxide ([U]SPIO). They consist of nonstoichiometric iron oxide cores (3-10 $\mathrm{nm}$ in diameter), whose composition and physicochemical properties vary continuously from magnetite $\mathrm{Fe}_{3} \mathrm{O}_{4}$ to maghemite $\gamma-\mathrm{Fe}_{2} \mathrm{O}_{3}$. For i.v. administration, they are generally synthesised in a one-step process by alkaline coprecipitation or iron (II) and iron (III) precursors in aqueous solutions of hydrophilic macromolecules, essentially dextran [201]. The role of these macromolecular corona is the limitation of the magnetic core growth during the synthesis, their steric stabilization in water (and later in physiological medium) and in vivo the reduction of the opsonization process. These colloidal contrast agents would be more realistically described as made of several magnetic cores, more or less aggregated and embedded in the hydrophilic macromolecules. The overall hydrodynamic diameter, as measured by photon correlation spectroscopy, is largely higher than the magnetic core dimensions. Interactions between magnetic cores and macromolecules are weak (essentially Van der Waals and hydrogen interactions) [27] and generally prevent any efficient derivatization of dextran corona without macromolecule depletion [202]. Stability improvement may be achieved when dextran macromolecules are cross-linked in a second step for enhancing the mechanical entrapment of magnetic cores (cross-linked iron oxide [CLIO] [28]) or when dextran macromolecules are chemically bonded to magnetic cores through the use of coupling agents [29] (versatile USPIO [VUSPIO] [30]). 
These very last generations of (U)SPIO contrast agents have, up to now, been essentially developed in academic laboratories.

(U)SPIO pharmacokinetics, toxicity and biodistribution properties were studied and allowed to define the potential uses of these contrast agents [31-35]. The lethal dose $\left(\mathrm{LD}_{50}\right)$ of the dextran-iron oxide complex was found to be $2000-6000 \mathrm{mg}_{\mathrm{Fe}} / \mathrm{kg}$ of body, whereas it is $300-600 \mathrm{mg}_{\mathrm{Fe}} / \mathrm{kg}$ for pristine iron oxide [35]. The nanoparticles are metabolized in lysosomes and the soluble iron becomes part of the normal iron pool (e.g., ferritin, hemosiderin, transferritin and hemoglobin) [26,36]. The iron load resulting from administration of the clinical dose ( $1 \mathrm{mg} \mathrm{Fe} / \mathrm{kg}$ of body) is low compared with the total store in the human body $(\sim 3500 \mathrm{mg})$.

Indeed, biodistribution and contrast enhancement are size-dependent. Larger nanoparticles (SPIO) exhibit a high $\mathrm{R}_{2} / \mathrm{R}_{1}$ relaxivity ratio and, because of their overall size ( $>40 \mathrm{~nm}$ in diameter), they are efficiently accumulated in MPS organs ( $20 \%$ of the injected dose in liver and $5-10 \%$ in the spleen with plasma half-life lower than $10 \mathrm{~min})$. Therefore, SPIO decrease liver and spleen signal within several minutes of i.v. administration $[37,38]$. Malignant tumors or metastases, which are typically devoid of a substantial number of phagocytic cells, appear as hyperintense (bright) lesions contrasted against the hypointense (black) liver on $\mathrm{T}_{2}$-weighted sequences (Figure 2).

USPIO, also called monocristalline iron oxide nanocompound (MION) by some authors, exhibits an overall hydrodynamic diameter lower than $40 \mathrm{~nm}$. Owing to their small size and the hydrophilicity of their dextran corona, they act as stealth particles. Their plasma half-life is higher than $20 \mathrm{~h}$ and therefore they remain in

Figure 2. Axial $\mathrm{T}_{2}$-weighted sequence of human liver with signal metastasis in segment 6 .
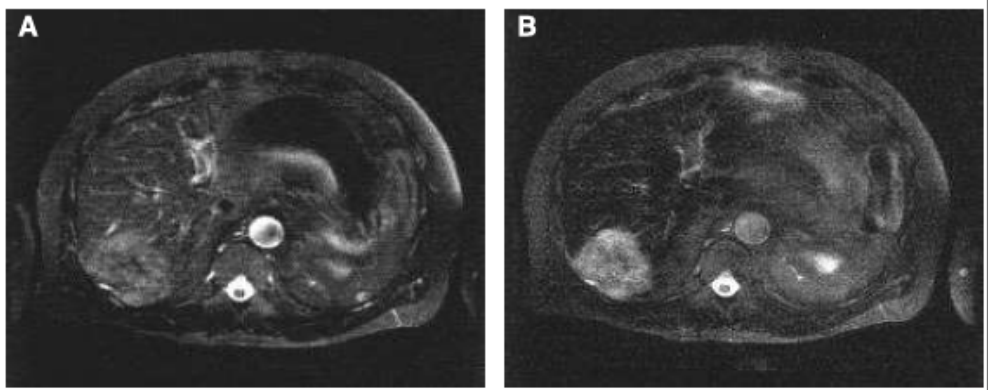

(A) Before and (B) following infusion of Endorem ${ }^{\circledR}$ (Guerbet, France). Reprinted with permission from [92]. the blood long enough to act as blood-pool agents for MR angiography (MRA). Some particles leak to the interstitium, where they are cleared by the macrophages of the lymphatic system or are drained via the lymphatic system and subsequently accumulated in the lymph nodes [39]. Thus, they can be used to diagnose hyperplastic and tumorous lymph node by MR lymphography [40,41]. A decrease in signal intensity indicates active uptake of particles into macrophages in normally functioning nodes, whereas an increase in signal intensity indicates altered capillary permeability in tumor (Figure 3). In comparison with SPIO, USPIO exhibit lower relaxivities, although the lower $R_{2} / R_{1}$ ratio leads to a higher $\mathrm{T}_{2}$ effect on $\mathrm{T}_{2}$-weighted images. However, USPIO have a much more favorable $\mathrm{T}_{1} / \mathrm{T}_{2}$ ratio that facilitates MRA or other $\mathrm{T}_{1}$-weighted $\mathrm{MR}$ techniques at low $\mathrm{B}_{0}$ (lower than $0.5 \mathrm{~T}$ ). Thus, USPIO significantly shorten the $T_{1}$ of blood during the first hours after i.v. injection and may be used as a $T_{1}$-agent [42].

Currently, molecular MRI is the main development in progress because clinicians hope for a contrast agent that would accumulate highly and specifically in malignant tumors, allowing an accurate diagnosis at a stage when the disease would be still treatable. Such tools would also be of a great interest for cardiovascular, inflammatory and degenerative diseases. Tissue-specific MR contrast agents have been reviewed recently $[36,43]$. Ligandlabeled superparamagnetic nanoparticles were designed (e.g., human polyclonal IgG [44], anticarcinoembryonic antigen [45], anti-glioma [46], L6 antibody [47] and anti-epidermal growth factor receptor [48]) and investigations in small animals revealed that it is possible to achieve a distinct concentration of the magnetic label at the target. However, the required dose of the labeled antibody is still too high to make a commercial development realistic [36]. As has been already discussed about active targeting in drug delivery, folate mediation also appears to be promising as a tumor MRI diagnostic since folate receptors are commonly overexpressed onto the surface of human cancer cells [49-52]. Since overexpression of endogenous transferring receptor ( $\mathrm{TfR}$ ) has been qualitatively described for various cancers and is presumably due to malignant transformation of cells, TfR may also represent a suitable target for the application of molecular MRI to increase detection of smaller tumors [53]. Thanks to the CLIO platform technology, quantitative measurement of $\mathrm{TfR}$ overexpression in a panel of 27 human breast cancer patients demonstrated that 


\section{Figure 3. MRI nodal abnormalities in two patients with} prostate cancer.

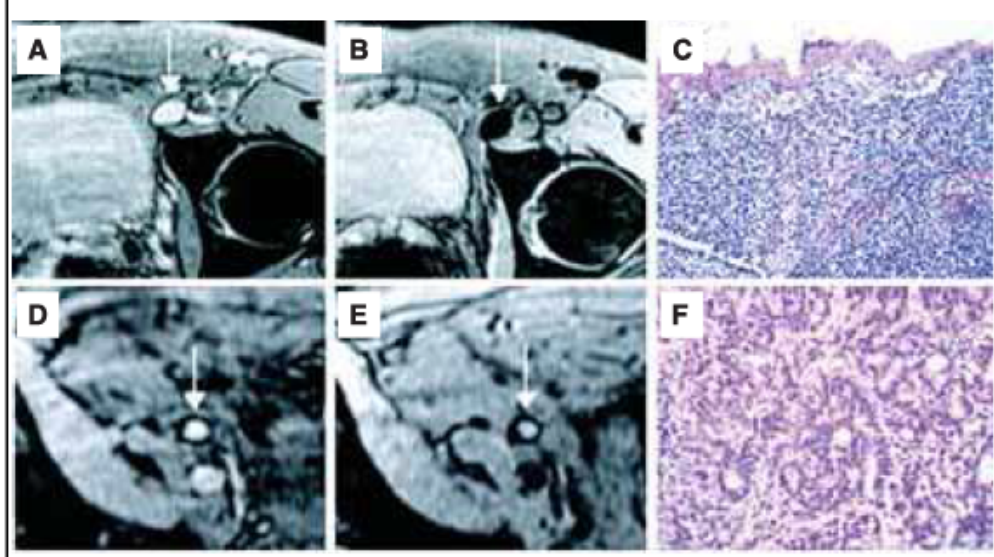

As compared with conventional MRI (A), MRI obtained $24 \mathrm{~h}$ after the administration of lymphotropic superparamagnetic nanoparticles (B) shows a homogeneous decrease in signal intensity owing to the accumulation of lymphotropic superparamagnetic nanoparticles in a normal lymph node in the left iliac region (arrow). (C) Shows the corresponding histological findings (hematoxylin and eosin, x125). Conventional MRI shows high signal intensity in an unenlarged iliac lymph node completely replaced by tumor (arrow in $\mathbf{D}$ ). Nodal signal intensity remains high (arrow in E). (F) Shows the corresponding histological findings (hematoxylin and eosin, x200).

Reprinted with permission from [93].
$74 \%$ of patient cancer tissues overexpressed the $\mathrm{TfR}$ and that the sensitivity of the new imaging agent was suitable to detect TfR overexpression in greater than $40 \%$ of these cases.

Last, the development and validation of MRI as a technique to rapidly screen compounds that are potential MR agents for their interaction with specific cellular targets was reported [54]. It was demonstrated that MRI can evaluate thousands of samples simultaneously and rapidly, provide exceedingly accurate measurements and provide receptor binding/internalization data as validated by radioactive assays. The technique allows the screening of libraries of peptide-nanoparticle conjugates against target cells and the identification of conjugates that may be used subsequently as reporter agents in vivo. The technology should greatly accelerate the development of target-specific or cell-specific MR contrast agents.

\section{Magnetic nanoparticles as mediators for magnetic hyperthermia}

The notion of hyperthermia (Greek word for overheating) in the treatment of a large variety of diseases and dysfunctions is as old as medicine itself [55]. Presenly, hyperthermia remains a promising form of cancer therapy aside from the well known methods of surgery, chemotherapy and radiotherapy. Clinical experiments taking advantage of the higher sensitivity of tumor cells to temperatures in the range of $42-45^{\circ} \mathrm{C}$ than normal tissue cells were reported in the 1970 s [56]. It is thought that, in such a temperature range, the function of many structural and enzymatic proteins within cells are modified, which, in turn, alters cell growth and differentiation and can induce apoptosis [57]. Thermoablation $\left(46-56^{\circ} \mathrm{C}\right)$ leads to tumor destruction by direct cell necrosis, coagulation or carbonization [58]. Nevertheless, the risk of overheating surrounding safe tissue necessitates an accurate temperature control, which is currently unrealistic. That is why the most widespread strategy is 'mild' hyperthermia, performed between 41 and $46^{\circ} \mathrm{C}$ in combination with other treatment modalities, such as chemotherapy or irradiation. The latter approach is based on growing knowledge regarding the microenvironmental conditions within the tumor; in particular, the fact that hypoxic (poorly oxygenated) cancerous cells are much more resistant to radiation than euoxic (well oxygenated) cancerous cells, whereas hypoxic cells are more heat-sensitive than euoxic cells [59]. Therefore, in order to obtain the greatest efficiency in cancerous cell destruction it appears logical to combine these two modalities of cancer therapy. Clinical results demonstrate that the combination of radiation therapy and hyperthermia resulted in a substantial therapeutic improvement [60]. One possible explanation of this combined effect is that heat induces malfunction of repair processes after radiation-induced DNA damage in cancerous cells [58]. Hyperthermia benefits were also reported for various diseases, such as gonoccal infection and syphilitic paralysis, and are also expected for HIV infection [61].

Current modalities for cancer hyperthermia may be classified according to the nature of the heating source and the heated target, from whole-body to tumoral cell level $[6,57,62,63]$. Main heating sources fall into three categories: contact with externally heated liquid, contactless applicator (e.g., ultrasound, microwave, radiofrequency and infrared devices) and inserted heating source (e.g., probes, antennas, laser fibers and mediators). Among the most recent hyperthermia devices, those that are based on focused ultrasound or electromagnetic radiation are available commercially. Nevertheless, none of these devices is able to accurately deliver high heat energy to deeply situated cancers without destroying the surrounding normal tissues, leading to the parallel development of technologies based on inserted heating sources. 
Among them, inductive mediators made of magnetic materials are more particularly developed because not only are they capable of converting the electromagnetic energy into heat when exposed to an external alternating current (AC) magnetic field, but tissues do not contain intrinsic magnetic matter [62]. With respect to the patient's comfort, it was found that the product H. $v$ (where $\mathrm{H}$ is the amplitude and $v$ the frequency of the $\mathrm{AC}$ magnetic field) should be lower than $4.85 \times 10^{8} \mathrm{Am}^{-1} \mathrm{~s}^{-1}$ for a treatment duration of $1 \mathrm{~h}$ [64]. Moreover, the frequency has to be greater than $50 \mathrm{kHz}$ to avoid neuromuscular electrostimulation and lower than $10 \mathrm{MHz}$ for appropriate penetration depth of the rf-field [65].

Interstitial macroscopic mediators for magnetic hyperthermia are generally ferromagnetic rods or seeds inserted directly into tumor tissues [63]. Their main limitations are the stressful surgical intervention, the difficult accessibility to some tumors, the potential thermoseed migration, the nonuniform temperature pattern and, therefore, the potential thermal underdosage of critical regions.

The latest magnetic hyperthermia modalities are based on micro- or nano-scale mediators in the form of injectable colloidal dispersion of magnetic particles and may be performed according to three strategies: arterial embolization hyperthermia $(\mathrm{AEH})$, direct injection hyperthermia $(\mathrm{DIH})$ and intracellular hyperthermia (IH) [6-63].

The origin(s) of magnetic particle heating essentially depends on their size and (hence) their magnetic properties $[6,13,64]$. For the largest particles (i.e., multidomain ferro- or ferrimagnetic materials), heating is due to hysteresis losses. In single-domain particles (superparamagnetic particles), magnetic energy is dissipated when the particle moment relaxes to its equilibrium orientation (Néel relaxation). Last, for both types of particles, heating can also be due to the rotational Brownian motion within a carrier liquid, that is, the rotation of the magnetic particle as a whole owing to the torque exerted on the magnetic moment by the external AC magnetic field. In this case, the energy barrier for reorientation of a particle is determined by rotational friction within the surrounding liquid (Brown relaxation). The efficiency of the particles is defined as the power of heating of a magnetic material per gram (specific absorption rate $[\mathrm{SAR}])$. SAR values depend on a large number of parameters (e.g.,size, size distribution, shape, bulk and surface chemical compositions, frequency and amplitude of the magnetic field viscosity of the surrounding medium) and vary from a few tenths to a few hundreds of Watt per gram of magnetic materials [6]. For clinical purposes, these values are crucial because the higher the SAR, the lower the injected dose to the patient. Presently, SPIO nanoparticles appear to be the best compromise solution combining biocompatibility, adequate SAR values and stable dispersion in physiological medium. Magnetoliposomes obtained by coating phospholipid onto magnetite particles were also investigated [66].

Since the pioneering work of Gilchrist and colleagues in 1957, magnetic hyperthermia has been the aim of numerous in vitro and in vivo investigations [63]. Currently, only Jordan's group in Berlin has reached the stage of clinical trials. They developed simultaneously the first fullsized human magnetic field applicator (MFH Hyperthermiesystem $\mathrm{GmbH}$, Berlin) [67,101] and a biocompatible magnetic fluid (MagForce Applications $\mathrm{GmBH}$, Berlin) made of aminated magnetite nanoparticles [203]. They validated their DIH approach in cell lines [68,69] as well as in experimental tumors (e.g., C3H mammary carcinoma [70] and orthotopic Dunning R3327 prostate tumor [71]). The first report of a clinical application dealt with the pilot treatment of prostate carcinoma in a patient aged 67 years with biopsy-proven local tumor progression following high-dose rate brachytherapy [72]. The nanoparticle dispersion was injected transperineally within the tumor and the number and the position of the depots were calculated according to the SAR value and the computerized tomography of the prostate. It appeared that nanoparticles were retained in the prostate for at least 6 weeks, allowing for repeated effective heat treatments at $4-5 \mathrm{kA} / \mathrm{m}(100 \mathrm{kHz})$. The aim to obtain sufficient temperatures in the prostate was achieved and the next step will be to initiate a Phase I study to evaluate feasibility, toxicity and quality of life during hyperthermia sequences. Several clinical studies are currently in progress [101]. Promising DIH experiments were also performed in animal models by using magnetite cores embedded within cationic liposomes [10,73-75].

One of the next challenges will be to develop the $\mathrm{IH}$ route, which is based on i.v. administered stealth magnetic nanoparticles designed for selective uptake by tumor cells and which would be the optimal method allowing tumor cells to be overheated selectively, even in disseminated metastases in any region of the body. However, at least three problems must be overcome first. 
The first issue is the ligand-mediated targeting to improve the colloidal mediator uptake by cancer cells. Among several in vitro studies [52,76], a single paper reported the use of ${ }^{111}$ In-chimeric L6 monoclonal antibodies grafted onto dextrancoated iron oxide cores [77]. After i.v. injection in tumor-bearing mice, the nanoparticles were preferentially uptaken in liver rather than in tumors, suggesting that, currently, this method is probably no better than DIH.

The second issue is the tissue distribution monitoring prior to the heating sequence. MRI appears to be the most suited method and, therefore, great interest exists to investigate a unique and versatile magnetic device that will be able to reveal tumors and metastases and treat them by hyperthermia within minutes.

The last issue concerns the control of in vivo temperature evolution during hyperthermia because, on the one hand, heat conduction and energy adsorption in vivo are widely unknown and, on the other hand, local overheating may damage safe tissue. This problem could be solved by monitoring the temperature via noninvasive means, such as MRI, owing to the temperaturedependence of proton relaxation times. This strategy is currently investigated in focused ultrasound hyperthermia where fast MRI proved to be very efficient for continuous temperature mapping and automatic feedback control of the ultrasound output [78]. It is not obvious that colloidal magnetic mediators do not disturb MR signals and allow accurate temperature maps. Another route could exploit the temperature dependence of magnetic properties. Indeed, Tc is the temperature at which ferro- and ferri-magnetic particles lose their magnetic properties, thus, they do not convert electromagnetic energy into heat. Therefore, $T_{c}$ is the maximal temperature reachable by magnetic particles. Choosing an appropriate Tc would be the smartest way to control hyperthermia because, in that case, particles would be both heaters and fuses. Such a strategy has already been developed for alloy thermoseeds in order to prevent local tissue overheating and reduce the need for invasive thermometry [79]. The design of colloidal mediators was reported recently: $\mathrm{La}_{0.8} \mathrm{Sr}_{0.2} \mathrm{MnO}_{3}$ and $\mathrm{La}_{0.75} \mathrm{Sr}_{0.25} \mathrm{MnO}_{3}$ particles were synthesized by the freeze-drying method and their size was reduced by high-energy planetary ball mill to $100-200 \mathrm{~nm}$ [80]. In AC magnetic field $(800 \mathrm{kHz}, 7.2 \mathrm{kA} / \mathrm{m})$, the maximal temperature reached by $\mathrm{La}_{0.75} \mathrm{Sr}_{0.25} \mathrm{MnO}_{3}$ particles was $46.3^{\circ} \mathrm{C}$ $\left(\mathrm{Tc}=56^{\circ} \mathrm{C}\right.$ ) and $37.8^{\circ} \mathrm{C}$ for $\mathrm{La}_{0.8} \mathrm{Sr}_{0.2} \mathrm{MnO}_{3}$ particles $\left(\mathrm{T}_{\mathrm{C}}=48^{\circ} \mathrm{C}\right)$. The difference between $\mathrm{T}_{\mathrm{c}}$ and the observed maximal temperature was assigned to the sharp decrease of saturation magnetization (usual for ferromagnetic materials in the vicinity of Tc and predicted by the ferromagnetic exchange theory) and to the heat exchange balance. Such results were corroborated in a more recent study where $\mathrm{La}_{0.75} \mathrm{Sr}_{0.25} \mathrm{MnO}_{3}$ particles were obtained in the $60-\mathrm{nm}$ range [81].

Last, a crucial topical question remains: is intracellular hyperthermia superior to extracellular hyperthermia? According to a theoretical model, there is no hyperthermic effect at the nanometric scale (particle size) or at the micrometric scale (cell size) [82]. Hyperthermia is only possible on a millimetric scale (tumor size), the isolating behavior of the cell membrane being negligable. If any experimental difference is observed between intracellular and extracellular hyperthermia, it can only be owing to 'chemical effects triggered by the presence of the nanoparticles or mechanical damage caused to the cell by intracellular vibrations and rotations of the nanoparticles'.

\section{Future perspectives: magnetic nanoparticles within carriers for drug delivery}

The development of pharmaceutical techniques that could deliver cytotoxic molecules selectively to the diseased site and with controlled kinetics is currently one of the most active areas of cancer research [8]. Magnetic particles could become innovative tools for controlling the drug biodistribution in space and time.

The principle of the magnetic guidance of drug delivery systems is based on submicronic particles containing simultaneously magnetic nanoparticles and drug molecules. When the particles have entered the bloodstream, external high-gradient magnetic fields are used to concentrate them at a specific target site within the body [13]. Such a strategy was developed several decades ago. The main difficulties to overcome were the stealthiness towards the MPS, the in vivo aggregation of the particles and the inherent risk of embolization, the development of strong enough magnets for the targeting of deep tissues and the disappointing results obtained in larger animal tests [7]. A Phase I clinical study was reported a few years ago and concerned 14 patients with advanced unsuccessfully treated cancers or sarcomas [83]. The magnetic carriers were starch particles $(50-150 \mathrm{~nm})$ containing magnetic cores of iron oxyhydroxides and capable of adsorbing antitumoral drugs. Although the responses to the treatment in most patients were rather low, the magnetic targeting 
was well tolerated, evidence of the accumulation of carriers in the tumor mass was given and relatively light side effects were noticed. The next challenges are a better knowledge of the hydrodynamics/field gradients correlation, the design of carriers of higher drug capacity, the combined use of specific ligands for increasing the targeting efficacy and retaining the particles in the vicinity of the target after removal of the external magnet and an accurate control of the drug release.

Concerning this last requirement, the drug release could also be triggered remotely thanks to the magnetic properties of the particles. A first strategy consisted of the application of an oscillating magnetic field, leading the particles to oscillate and the drug to be released in these mechanical stress conditions [84]. For instance, the release rate of insulin may be 50 -times higher than in the absence of the magnetic field. A second strategy could take advantage of the ability of magnetic nanoparticles to heat in AC magnetic fields (see hyperthermia section). The temperature increase could greatly accelerate the drug diffusion across the carrier matrix. In the case of a thermosensitive hydrogel matrix (e.g., copolymers based on $\mathrm{N}$-isopropylacrylamide units), it could suddenly collapse the structure causing a 'burst' release of the carried drug. As far as we know, such a device has not yet been reported with magnetic particles. Although a parallel strategy based on gold colloids able to heat under the light of a pulsed near-IR laser was reported a few years ago [85].

\section{Conclusions}

Iron oxide magnetic nanoparticles are now used routinely as contrast agents in MRI and new applications are expected in the field of cancer thermal treatment, magnetic targeting and remotely triggered drug release. These devices will be nanomaterials of higher and higher added value, since they are expected to be multifunctional, smart and versatile. Therefore, their chemical composition shall become more and more hybrid, combining inorganic, organic and biological components.

For a complete overview of their potential applications, it should be noted here that magnetic particles are also being developed for other uses, such as cell sorting $[7,11]$, detoxification of biological fluids [12], radionucleide and gene delivery (magnetofection) $[7,12,13]$, retinal detachment [11], cell membrane manipulation $[8,12]$ and magnetorelaxometry [11].

Such developments of medicine-directed nanotechnology are probably among the best examples where physicians, pharmacologists, biologists, chemists and physicists are working together. At present, when public opinion rightly raises concerns regarding the possible consequences of nanotechnology on mankind and its environment, it is important to highlight that the single aim of the majority of these research efforts on nanoparticles is the survival and comfort of humanity.

\section{Acknowledgements}

The authors' work was performed, in part, in the framework of the European Network of Excellence Functional Advanced Materials and Engineering (FAME): Ceramics and Hybrids. The authors wish to thank Jacques E Bonnet, Anne-Karine Bouzier-Sore, Valérie Cabuil, Jean-Marie Caillé, Paul Canioni, Patrick Couvreur, Mathilde Deloire-Grasin, Marie Hélène Delville, Alain Demourgues, Martine Dorian, Vincent Dousset, Catherine Dubernet, Michel Fontanille, Léopold Fournès, Jean-Michel Franconi, Graziella Goglio, Valérie Heroguez, Christine Labrugère, Stéphanie Lassiaz, Sylvain Miraux, Klaus Petry, Emil Pollert, Josik Portier, Lydia Raison, Fabio Sonvico, Eric Thiaudière, Matthieu Valle, Antoine Vekris, Pavel Veverka, Pierre Voisin and Alain Wattiaux for valuable collaboration and helpful discussions.

Executive summary

\section{Introduction}

- Owing to their size of a few nanometers, inorganic nanoparticles are capable of displaying unusual physical properties, to diffuse through biological membranes and interact closely with biomolecules. Magnetic nanoparticles are of particular interest for enhancing MRI contrast, heating tumors at the cellular level and guiding drugs to their target.

\section{Magnetic nanoparticle design}

- Magnetite $\mathrm{Fe}_{3} \mathrm{O}_{4}$ and maghemite $\gamma-\mathrm{Fe}_{2} \mathrm{O}_{3}$ are by far the most commonly employed oxides, owing to their good chemical stability and biocompatibility.

- Prior to intravenous (i.v.) administration, they are encapsulated in a corona of hydrophilic macromolecules to avoid their uncontrolled aggregation and delay their clearance by the mononuclear phagocyte system.

- For active targeting, they are derivatized with ligands (oligosaccharides, oligopeptides, folic acid, antibodies and their fragments) that are expected to specifically bind to surface epitopes or receptors on the target sites, opening the possibility of targeting specific cell types or subsets of cells within the vasculature and even elements of vascular emboli and thrombi. 


\section{Executive summary}

\section{Magnetic nanoparticles as contrast agents for MRI}

- Ultrasmall superparamagnetic iron oxide (USPIO) are used currently in hospitals for MRI of liver or lymph nodes.

- Development of tissue-specific MR contrast agents is currently in progress (molecular MRI) since such agents would be of great interest for cardiovascular, inflammatory and degenerative diseases.

\section{Magnetic nanoparticles as mediators for magnetic hyperthermia}

- Magnetic nanoparticles are able to heat in an alternating current magnetic field. In oncology, a promising therapy would consist of inserting them essentially within the tumor mass in a homogeneous way.

- In Jordan's group in Berlin, clinical trials are currently in progress for a direct injection strategy.

- One of the next challenges will be to develop an intracellular hyperthermia route, which would be based on i.v. administered stealth magnetic nanoparticles designed for selective uptake by tumor cells and which would be the optimal method allowing tumor cells to be overheated selectively, even in disseminated metastases in any region of the body.

- At least three problems must be overcome first: the ligand-mediated targeting, the tissue distribution monitoring prior to and the control of in vivo temperature evolution during the heating sequence.

\section{Magnetic nanoparticles within carriers for drug delivery}

- A few years ago, drug magnetic guidance was the aim of a first Phase I clinical study. Evidence of the accumulation of carriers in the tumor mass were given.

- The next challenges are a better knowledge of the hydrodynamics/field gradients correlation, the design of carriers of higher drug capacity, the combined use of specific ligands for increasing the targeting efficacy and retaining the particles in the vicinity of the target after removal of the external magnet and accurate control of drug release.

- Other developments expect to take advantage of the ability of magnetic nanoparticles to vibrate or heat in AC magnetic fields for increasing the drug release kinetics (remotely triggered controlled release).

\section{Conclusion}

- At present, when public opinion rightly raises concerns about the possible consequences of nanotechnology on mankind and its environment, it is important to highlight that the single aim of the majority of nanotechnology research efforts is the survival and comfort of humanity.

\section{Bibliography}

1. Häfeli U: The history of magnetism in medicine. In: Magnetism in Medicine. Andrä W, Nowak H (Eds). Wiley-VCH, Berlin, Germany, 15-34 (1998).

2. Bailey RE, Smith AM, Nie S: Quantum dots in biology and medicine. Physica E25, 1-12 (2004).

-3. West JL, Halas NJ: Engineered nanomaterials for biophotonics applications: improving sensing, imaging, and therapeutics. Annu. Rev. Biomed. Eng. 5 , 285-292 (2003).

4. Moghimi SM, Szebeni J: Stealth liposomes and long circulating nanoparticles: critical issues in pharmacokinetics, opsonization and protein-binding properties. Prog. Lipid Res. 42(6), 463-478 (2003).

-5. Monfardini C, Veroneses FM: Stabilization of substances in circulation. Bioconjug. Chem. 9(4), 418-450 (1998).

6. Mornet S, Vasseur S, Grasset F et al: Magnetic nanoparticle design for medical diagnosis and therapy. J. Mater. Chem. 14, 2161-2175 (2004).

7. Sonvico F, Dubernet C, Colombo P et al.: Metallic colloid nanotechnology, applications in diagnosis and therapeutics. Curr. Pharm. Des. 11, 2091-2105 (2005).
8. Berry CC, Curtis ASG: Functionalisation of magnetic nanoparticles for applications in biomedicine. J. Phys. D Appl. Phys. 36, R198-R206 (2003).

9. Bahadur D, Giri J: Biomaterials and magnetism. Sadhana 28(3-4), 639-656 (2003).

10. Ito A, Shinkai M, Honda H et al: Medical application of functionalized magnetic nanoparticles. J. Biosci. Bioeng. 100(1), 1-11 (2005).

11. Tartaj P, del Puerto Morales M, Veintemillas-Verdaguer $S$ et al:: The preparation of magnetic nanoparticles for applications in biomedicine. J. Phys. D Appl. Phys. 36(13), R182-R197 (2003).

12. Gupta AK, Gupta MP: Synthesis and surface engineering of iron oxide nanoparticles for biomedical applications. Biomaterials 26, 3995-4021 (2005).

13. Pankhurst QA, Connolly J, Jones SK et al:: Applications of magnetic nanoparticles in biomedicine. J. Phys. D Appl. Phys. 36, R167-R181 (2003).

14. Berkov D: Basic physical principles. In: Magnetism in Medicine. Andrä W, Nowak H (Eds). Wiley-VCH, Berlin, Germany, 35-73 (1998).
15. Moghimi SM, Hunter AC, Murray JC: Long-circulating and target-specific nanoparticles: theory to practice. Pharmacol. Rev. 53(2), 283-318 (2001).

16. Hume DA, Ross IL, Himes SR et al:: The mononuclear phagocyte system revisited. J. Leukoc. Biol. 72, 621-627 (2002).

17. Chiannilkulchai N, Driouich Z, Benoit JP et al: Doxorubucin loaded nanoparticles: increased efficiency in murine hepatic metastases. Sel. Cancer Ther. 5, 1-11 (1990).

18. Pinto-Alphandary H, Andremont A, Couvreur P: Targeted delivery of antibiotics using liposomes and nanoparticles: research and applications. Int. J. Anitmicrob. Agents 13, 155-168 (2000)

19. Choi SW, Kim WS, Kim JH: Surface modification of functional nanoparticles for controlled drug delivery. J. Dispersion Sci. Technol. 24(3-4), 475-487 (2003).

20. Zalipsky S: Functionalized poly(ethylene glycol) for preparation of biologically relevant conjugates. Bioconjug. Chem. 6(2), 150-165 (1995).

21. Peracchia MT: Stealth nanoparticles for intravenous administration. STP Pharma. Sci. 13(3), 155-161 (2003). 
22. Weissleder R, Bogdanov A, Neuwelt EA et al: Long-circulating iron oxides for MR imaging. Adv. Drug Deliv. Rev. 16(2-3), 321-334 (1995).

23. Sudimack J, Lee RJ: Targeted drug delivery via the folate receptor. Adv. Drug Deliv. Rev. 41, 147-162 (2000).

24. Bonnemain B: Superparamagnetic agents in magnetic resonance imaging: physicochemical characteristics and clinical applications a review. J. Drug Target. 6(3), 167-174 (1998).

25. Bjornerud A, Johansson L: The utility of superparamagnetic contrast agents in MRI: theoretical consideration and applications in the cardiovascular system. NMR Biomed. 17 . 465-477 (2004).

26. Okuhata Y: Delivery of diagnostic agents for magnetic resonance imaging. Adv. Drug Deliv Rev. 37, 121-137 (1999).

27. Jung CW: Surface properties of superparamagnetic iron oxide MR contrast agents: ferumoxides, ferumoxtran, ferumoxsil. Magn. Reson. Imaging 13(5), 675-691 (1995).

28. Högemann D, Josephson L, Weissleder R et al: Improvement of MRI probes to allow efficient detection of gene expression. Bioconjug. Chem. 11(6), 941-946 (2000).

29. Lesniak C, Schiestel T, Nass R et al: Synthesis and surface modification of deagglomerated superparamagnetic nanoparticles. Mater. Res. Soc. Symp. Proc. 432, 169-174 (1997).

30. Mornet S, Portier J, Duguet E: A method for synthesis and functionalization of ultrasmall superparamagnetic covalent carriers based on maghemite and dextran. J. Magn. Magn. Mater. 293, 127-134 (2005).

31. Weissleder R, Stark DD, Engelstad BL et al: Superparamagnetic iron oxide: pharmacokinetics and toxicity. AJR Am. J. Roentgenol. 152, 167-173 (1989).

32. Papisov MI, Bogdanov A, Schaffer B et al: Colloidal magnetic resonance contrast agents: effect of particule surface on biodistribution. J. Magn. Magn. Mater. 122, 383-386 (1993)

33. Weissleder R, Elizondo G, Wittenberg J et al: Ultrasmall superparamagnetic iron oxide: characterization of a new class of contrast agents for MR imaging. Radiology 175 , 489-493 (1990).

-34. Brusentsov NA, Gogosov VV, Brusentsova TN et al: Evaluation of ferromagnetic fluids and suspensions for the site-specific radiofrequency-induced hyperthermia of MX11 sarcoma cells in vitro. J. Magn. Magn. Mater. 225, 113-117 (2001).

35. Wada S, Yue L, Tazawa K et al: New local hyperthermia using dextran magnetite complex (DM) for oral cavity: experimental study in normal hamster tongue. Oral Dis. 7, 192-195 (2001).
36. Weinmann HJ, Ebert W, Misselwitz B et al: Tissue-specific MR contrast agents. Eur. J. Radiol. 46, 33-44 (2003).

37. Reimer P, Balzer T: Ferucarbotran (Resovist): a new clinically approved RESspecific contrast agent for contrast-enhanced MRI of the liver: properties, clinical development and applications. Eur. Radiol. 13, 1266-1276 (2003).

38. Jung CW, Jacobs P: Physical and chemical properties of superparamagnetic iron oxide MR contrast agents: ferumoxides, ferumoxtran, ferumoxsil. Magn. Reson. Imaging 13(5), 661-674 (1995).

39. Weissleder R, Heautot JF, Schaffer BK et al: MR lymphography: study of highefficiency lymphotropic agent. Radiology 191, 225-230 (1994).

40. Guimaraes R, Clement O, Bittoun J et al:: MR lymphography with superparamagnetic iron nanoparticles in rats: pathologic basis for contrast enhancement. AJR Am. J. Roentgenol. 162, 201-207 (1994).

41. Bellin MF, Lebleu L, Meric JB: Evaluation of retroperitoneal and pelvic lymph node metastases with MRI and MR lymphangiography. Abdom. Imaging 28(2), 155-163 (2003).

42. Kellar KE, Fujii DK, Gunther WHH et al: "NC 100150", a preparation of iron oxide nanoparticles ideal for positive-contrast MR angiography. Magn. Reson. Mater. Phys. Biol. Med. 8(3), 207-213 (1999).

43. Bulte JWM, Kraitchman DL: Iron oxide MR contrast agents for molecular and cellular imaging. NMR Biomed. 17 , 484-499 (2004).

44. Weissleder R, Lee AS, Fischman AJ et al: Polyclonal human immunoglobin $\mathrm{G}$ labeled with polymeric iron oxide: antibody MR imaging. Radiology 181(1), 245-249 (1991).

45. Tiefenauer LX, Kühne G, Andres RY: Antibody-magnetite nanoparticles: in vitro characterization of a potential tumorspecific contrast agent for magnetic resonance imaging. Bioconjug. Chem. 4 , 347-352 (1993).

46. Suzuki M, Honda $\mathrm{H}$, Kobayashi $\mathrm{T}$ et al:: Development of a target-directed magnetic resonance contrast agent using monoclonal antibody-conjugated magnetic particles. Brain Tumor Pathol. 13, 127-132 (1996).

47. Remsen LG, McCormick CI, Roman-Goldstein $\mathrm{S}$ et al: $\mathrm{MR}$ of carcinoma-spectific monoclonal antibody conjugated to monocrystalline iron oxide nanoparticles : the potential for non invasive diagnostic. Am. J. Neuroradiol. 17(3), 411-418 (1996).
48. Suwa T, Ozawa S, Ueda M et al:: Magnetic resonance imaging of oesophageal squamous cell carcinoma using magnetite particles coated with anti-epidermal growth factor receptor antibody. Int. J. Cancer 75, 626-634 (1998).

49. Zhang Y, Kohler N, Zhang M: Surface modification of superparamagnetic magnetite nanoparticles and their intracellular uptake. Biomaterials 23, 1553-1561 (2002).

50. Kohler N, Fryxell GE, Zhang M: A bifunctional poly(ethylene glycol) silane immobilized on metallic oxide-based nanoparticles for conjugation with cell targeting agents. J. Am. Chem. Soc. 126(23), 7206-7211 (2004).

-51. Choi HC, Choi SR, Zhou R et al: Iron oxide nanoparticles as magnetic resonance contrast agent for tumor imaging via folate receptortarget delivery. Acad. Radiol. 11, 996-1004 (2004).

52. Sonvico F, Mornet S, Vasseur S et al: Folateconjugated iron oxide nanoparticles for solid tumor targeting as potential specific magnetic hyperthermia mediators: synthesis, physicochemical characterisation and in vitro experiments. Bioconjug. Chem. 16, 1181-1188 (2005).

53. Högemann-Savellano $\mathrm{D}$, Bos E, Blondet $\mathrm{C}$ et al:: The transferrin receptor: a potential molecular imaging marker for human cancer. Neoplasia 5(6), 495-506 (2003).

54. Högemann D, Ntziachristos V, Josephson L et al: High throughput magnetic resonance imaging for evaluating targeted nanoparticle probes. Bioconjug. Chem. 13, 116-121 (2002).

55. Nielsen OS, Horsman M, Overgaard J: A future for hyperthermia in cancer treatment? Eur. J. Cancer 37, 1587-1589 (2001).

56. Overgaard K, Overgaard J: Investigations on the possibility of a thermic tumour therapy - I. Eur. J. Cancer 8, 65-78 (1972).

57. Moroz P, Jones SK, Gray BN: Status of hyperthermia in the treatment of advanced liver cancer. J. Surg. Oncol. 77, 259-269 (2001).

58. Jordan A, Scholz R, Wust P et al:: Magnetic fluid hyperthermia (MFH): cancer treatment with $\mathrm{AC}$ magnetic induced excitation of biocompatible superparamagnetic nanoparticles. J. Magn. Magn. Mater. 201, 413-419 (1999).

59. Overgaard J: The current and potential role of hyperthermia in radiotherapy. Int. J. Radiat. Oncol. Biol. Phys. 16(3), 535-549 (1989).

60. Dubois JB: Hyperthermie: principes, techniques. Place actuelle dans le traitement des cancers. Bull. Cancer Radiother. 82 , 207-224 (1995). 
61. Owens SD, Gasper PW: Hyperthermic therapy for HIV infection. Med. Hypotheses 44, 235-242 (1995).

62. Gel'vich EA, Mazokhin VN: Technical aspects of electromagnetic hyperthermia in medicine. Crit. Rev. Biomed. Eng. 29(1), 77-97 (2001).

63. Moroz P, Jones SK, Gray BN: Magnetically mediated hyperthermia: current status and future directions. Int. J. Hyperthermia 18(4), 267-284 (2002).

64. Andrä W: Magnetic hyperthermia. In: Magnetism in Medicine. Andrä W, Nowak H (Eds). Wiley-VCH, Berlin, Germany, 455-470 (1998).

65. Hill DA: Further studies of human wholebody radiofrequency absorption rates. Bioelectromagnetics 6(1), 33-40 (1985).

66. Le B, Shinkai M, Kitade T et al: Preparation of tumor-specific magnetoliposomes and their application for hyperthermia. J. Chem. Eng. Jpn 34(1), 66-72 (2001).

-67. Gneveckow U, Jordan A, Scholz R et al:: Description and characterization of the novel hyperthermia- and thermoablationsystem MFH (R) $300 \mathrm{~F}$ for clinical magnetic fluid hyperthermia. Med. Phys. 31(6), 1444-1451 (2004).

68. Jordan A, Wust P, Scholz R et al: Cellular uptake of magnetic fluid particles and their effects on human adenocarcinoma cells exposed to AC magnetic fields in vitro. Int. J. Hyperthermia 12(6), 705-722 (1996).

-69. Jordan A, Scholz R, Wust P et al:: Endocytosis of dextran and silan-coated magnetite nanoparticles and the effect of intracellular hyperthermia on human mammary carcinoma cells in vitro. J. Magn. Magn. Mater. 194, 185-196 (1999).

70. Jordan A, Scholz R, Wust P et al:: Effects of magnetic fluid hyperthermia (MFH) on C3H mammary carcinoma in vivo. Int. J. Hyperthermia 13(6), 587-605 (1997).

71. Johannsen M, Jordan A, Scholz R et al:: Evaluation of magnetic fluid hyperthermia in a standard rat model of prostate cancer. J. Endurolog. 18(5), 495-500 (2004).

72. Johannsen M, Gneveckow U, Eckelt L et al: Clinical hyperthermia of prostate cancer using magnetic nanoparticles: presentation of a new interstitial technique. Int. J. Hyperthermia 21(7), 637-647 (2005).
73. Kawai N, Ito A, Nakahara Y et al: Anticancer effect of hyperthermia on prostate cancer mediated by magnetite cationic liposomes and immune-response induction in transplanted syngeneic rats. Prostate 64, 373-381 (2005)

74. Tanaka K, Ito A, Kobayashi T et al:: Intratumoral injection of immature dendritic cells enhances antitumor effect of hyperthermia using magnetic nanoparticles. Int. J. Cancer 116, 624-633 (2005).

-75. Kawai N, Ito A, Nakahara Y et al: Complete regression of esperimental prostate cancer in nude mice by repeated hyperthermia using magnetite cationic liposomes and a newly developed solenoid containing a ferrite core. Prostate 66, 718-727 (2006).

76. Suzuki M, Shinkai M, Kamihira M et al:: Preparation and characteristics of magnetitelabelled antibody with the use of poly(ethylene glycol) derivatives. Biotechnol. Appl. Biochem. 21, 335-345 (1995).

-77. DeNardo SJ, DeNardo GL, Miers LA et al.: Development of tumor targeting bioprobes (111 In-chimeric L6 monoclonal antibody nanoparticles) for alternating magnetic field cancer therapy. Clin. Cancer Res. 11, 7087s-7092s (2005).

78. Quesson B, Vimeux F, Salomir R et al: Automatic control of hyperthermic therapy based on real-time fourier analysis of MR temperature maps. Magn. Reson. Med. 47(6), 1065-1072 (2002).

-79. Deger S, Taymoorian K, Boehmer D et al: Thermoradiotherapy using interstitial selfregulating thermoseeds: an intermediate analysis of a phase II trial. Eur. Urol. 45(5), 574-579 (2004).

80. Kuznetsov AA, Shlyakhtin OA, Brusentsov NA et al:: "Smart" mediators for self-controlled inductive heating. Eur. Cell. Mater. 3(Suppl. 2), 75-77 (2002).

81. Vasseur S, Duguet E, Portier J et al:: Lanthanum manganese perovskite nanoparticles as possible in vivo mediators for magnetic hyperthermia. J. Magn. Magn. Mater. 302, 315-320 (2006).

82. Rabin Y: Is intracellular hyperthermia superior to extracellular hyperthermia in the thermal sense? Int. J. Hyperthermia 18(3), 194-202 (2002).

-83. Lübbe AS, Alexiou C, Bergemann C: Clinical applications of magnetic drug targeting. J. Surg. Res. 95, 200-206 (2001).
84. Edelman ER, Kost J, Bobeck T et al:: Regulation of drug release from polymer matrices by oscillating magnetic fields. J. Biomed. Mater. Res. 19, 67-83 (1985).

-85. Sershen SR, Westcott SL, Halas NJ et al: Temperature-sensitive polymer-nanoshell composites for photothermally modulated drug delivery. J. Biomed. Mater. Res. 51, 293-298 (2000).

86. Kreuter J: Nanoparticutate systems for brain delivery drugs. Adv. Drug Deliv. Rev. 47, 65-81 (2001).

-87. Lockman PR, Mumper RJ, Khan MA et al:: Nanoparticle technology for drug delivery across the blood-brain barrier. Drug Dev. Ind. Pharm. 28(1), 1-12 (2002).

88. Couvreur P, Brigger I, Dubernet C: Nanoparticles in cancer therapy and diagnosis. Adv. Drug Deliv. Rev. 54(5), 631-651 (2002).

89. Kumar MNV: Nano and microparticles as controlled drug delivery devices. J. Pharm. Pharm. Sci. 3(2), 234-258 (2000).

90. Ghosh PK: Hydrophilic polymeric nanoparticles as drug carriers. Indian J. Biochem. Biophys. 37, 273-282 (2000).

91. Mordon S, Desmettre T, Devoisselle JM et al: Selective laser photocoagulation of blood vessels in a hamster skin flap model using a specific ICG formulation. Laser Surg. Med. 21(4), 365-373 (1997).

92. Wallis F, Gilbert FJ: Magnetic resonance imaging in oncology: an overview. JR Coll. Surg. Edinb. 44, 117-125 (1999).

-93. Harisinghani MG, Barentsz J, Hahn PF et al: Non invasive detection of clinically occult lymph-node metastases in prostate cancer. N. Engl. J. Med. 348, 2491-2499 (2003).

\section{Website}

101. MagForce Nanotechnologies AG www.magforce.de/en/

\section{Patents}

201. CANADIAN PATENTS AND DEVELOPMENT LIMITED.: US4452773 (1984).

202. ADVANCED MAGNETICS, INC.: US5248492 (1993).

203. INSTITUT FUR NEUE MATERIALIEN GEM. GmbH: US6541039 (2003). 